\title{
Estimation of Emissions During Monitoring of Pipelines in the Dynamic Mode of Operation
}

\author{
Maria Zemenkova ${ }^{1, *}$, Yuri Zemenkov ${ }^{1}$, Alexey Gladenko ${ }^{2}$ and Sergey Podorozhnikov ${ }^{1}$ \\ ${ }^{1}$ Tyumen Industrial University, Volodarskogo st. 38,Tyumen, Russia \\ ${ }^{2}$ Omsk State Technical University, st. Mira 11, Omsk, Russia
}

\begin{abstract}
To determine and diagnose the emissions from pipelines operating in the dynamic mode, the model is developed in real time, whereby the determination of the oil leakages volume is possible with sufficient accuracy. The stated methods assume the use of computer equipment and software. The use of sensitive devices and automation of data processing make it possible to achieve high accuracy of calculations. The developed techniques are meant to operate in systems of support and decision-making and expert systems. The rapid assessment results can be used by supervisory agencies to evaluate the damage in case of accidents and failures of pipelines.
\end{abstract}

\section{Introduction}

The current emissions control systems on pipelines are based on different physical methods (acoustic, vibroacoustic, estimation methods of estimation of the insulating covering of the pipeline conductivity, using the fluorescence method, etc.) and provide a registration of large leakages inaccuracy up to $5 \mathrm{~km}$. The quality of the models and methods of processing the results of diagnostics is particularly important.

\section{Methods}

For determining and diagnosing leakages of pipelines operating in a dynamic mode, the models for estimating in real time were developed. With their help, it becomes possible to define the volume of oil leakage with sufficient accuracy $[1-4,7,10]$. The stated methods assume the use of computer equipment and sophisticated software with the use of sensitive devices and automation $[5,6,8,9]$. Depressurization of the pipeline immediately affects the regime parameters of work preceding and following pumping stations (Figure 1) [12-14]. Thus, if the normal operating mode of the pumping station and pipeline can be characterized by the operating point $\left(\mathrm{F}_{\mathrm{r} 0}, \mathrm{Q}_{0}\right)$, in violation of the integrity of the pipeline, Fr0 pressure during the time interval $\tau$ decreases to $\mathrm{Fr}$ and $\mathrm{Q}_{0}$ consumption will increase to $\mathrm{Q}$. In this case, the greater the damage is, the more significant changes of $\mathrm{Fr} 0$ and $\mathrm{Q}_{0}$ will take place. Therefore, is possible to judge on the leakage value by the degree change of regime characteristics. Suppose that $\mathrm{Q}_{0}, \mathrm{~F}_{\mathrm{r} 0}$ - oil rate and pressure in the initial section of

* Corresponding author:muzemenkova@mail.ru 
the pipeline under normal operating conditions of pumping stations [15-18], $Z_{B}$ and $Z_{E}-$ benchmark of the beginning and end of the pipeline, $\mathrm{L}$ - length of the pipeline (Figure 2.). There is also the pumping mode, i.e. $\beta$ and $\mathrm{m}$, the kinematical viscosity of the pumped product $v$ and pipe diameter $d$.

$$
i_{0}=\beta \frac{Q_{0}^{2-m} v^{m}}{d^{5-m}}
$$

The hydraulic gradient during normal operation of the pipeline can be determined by well-known Leibenson formula.

Suppose that at a distance $x_{a}$ from the first pumping station (PSI) depressurization of the pipeline happened [19-20]. Benchmark of point of fault is $Z_{a}$. Pumping stations due to the inertia of registering devices and executive mechanisms have been working for some time $\tau$. The length of time $\tau$ can be divided into shorter periods of time $\Delta \tau_{j}$, and for each of these periods of time can determine flow rate $Q_{X}(\tau)$ in the pipeline section to the point of fault $x_{a}$ by operating point of the pump station and pipeline (see Figure 1).

Leakage rate can be found as the difference between the flow rate $Q_{x}(\tau)$ and flow rate $Q_{L}(\tau)$ in the area $\left(L-x_{a}\right)$, i.e.:

$$
Q_{y}(\tau)=Q_{x}(\tau)-Q_{L}(\tau)
$$

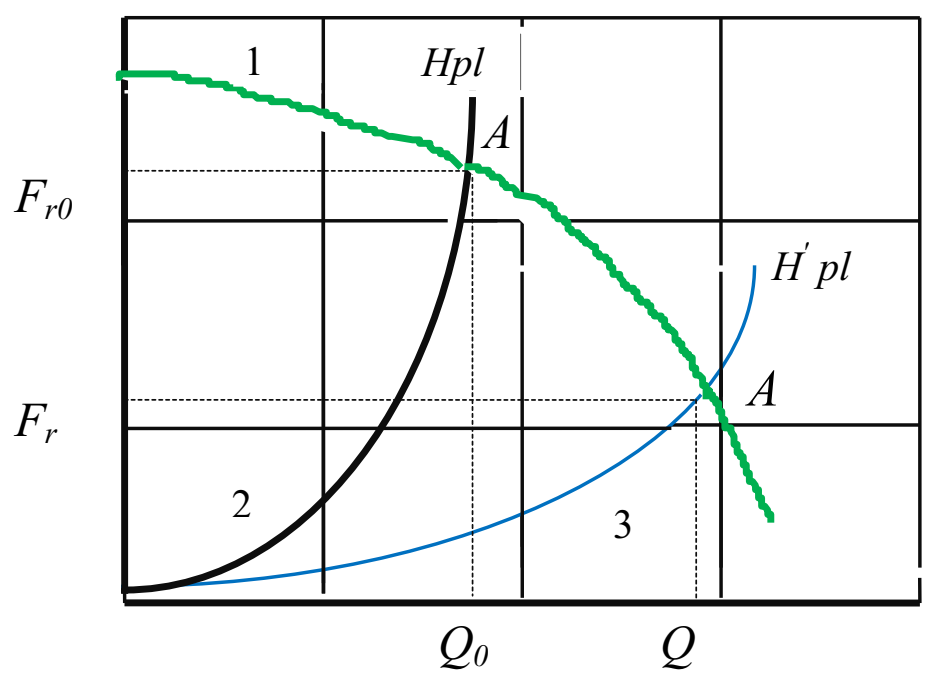

Fig. 1. Change the hydraulic parameters of the pipeline in its depressurization (HPL and $\mathrm{H}^{\prime} \mathrm{PL}$ pipeline characteristics before and after the accident). 


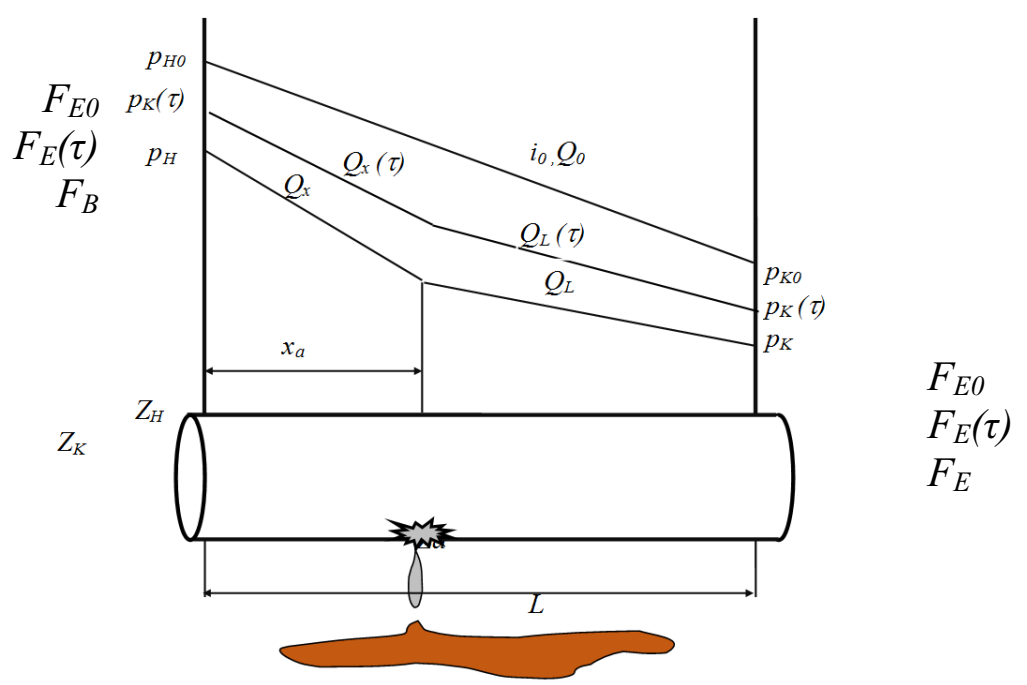

Fig. 2. Measurements of hydraulic parameters of the pipeline in violation of its depressurization (change of hydraulic gradient).

Consumption $\mathrm{Q}_{\mathrm{L}}(\tau)$ can be determined using the ratio of hydraulic ramps $i_{o}$ and $i_{L}(\tau)$, determined by the generalized formula Leibenson. Here, it is assumed that $\beta_{o}=\beta_{L}$ and $m_{o}$ $=m_{L}$ :

$$
Q_{L}(t)=Q_{0} \cdot\left(\frac{i_{L}(\tau)}{i_{0}}\right)^{\frac{1}{2-m}}
$$

where $i_{L}(\tau)$ - hydraulic gradient at the site $(L-x a)$.

To determine the hydraulic gradient $i_{L}(\tau)$, draw up Bernoulli equation for the area $x_{a}$ at working pipe station with a damaged pipeline

$$
Z_{H}+\frac{p_{H}(\tau)}{\rho g}+\frac{\vartheta_{H}^{2}(\tau)}{2 g}=Z_{a}+\frac{p_{a}(\tau)}{\rho g}+\frac{\vartheta_{a}^{2}(\tau)}{2 g}+i_{X}(\tau) \cdot x_{a}
$$

where $p_{a}(\tau)$ and $\vartheta_{a}(\tau)$ - the pressure and flow rate of the product before failure in the $\mathrm{j}$ th time. Since $\vartheta_{H}(\tau)=\vartheta_{a}(\tau)$, then we have

$$
Z_{a}+\frac{p_{a}(\tau)}{\rho g}=Z_{H}+\frac{p_{H}(\tau)}{\rho g}-i_{X}(\tau) \cdot x_{a}
$$

Bernoulli's equation for the pipeline section $(L-x a)$ :

$$
Z_{a}+\frac{p_{L}(\tau)}{\rho g}+\frac{\vartheta^{2}(\tau)}{2 g}=Z_{K}+\frac{p_{K}(\tau)}{\rho g}+\frac{\vartheta^{2}{ }_{K}(\tau)}{2 g}+i_{L}(\tau) \cdot\left(L-x_{a}\right)
$$

where $p_{L}(\tau)$ and $\vartheta_{L}(\tau)$ - the pressure and flow rate of the transported product after injury in $\mathrm{j}$ - th time.

Considering that section $(L-x a)$ also $\vartheta_{L}(\tau)=\vartheta_{K}(\tau)$ and substituting (5) into (6), we get 


$$
Z_{H}+\frac{p_{H}(\tau)}{\rho g}+i_{X}(\tau) \cdot x_{a}=Z_{K}+\frac{p_{K}(\tau)}{\rho g}+i_{L}(\tau) \cdot\left(L-x_{a}\right)
$$

Hydraulic gradient $i_{x}(\tau)$ can be obtained in similar way to (3):

$$
i_{X}(\tau)=i_{0} \cdot\left(\frac{Q_{X}(\tau)}{Q_{0}}\right)^{2-\mathrm{m}}
$$

Then (8) can be represented as following:

$$
Z_{H}+\frac{p_{H}(\tau)}{\rho \cdot g}-i_{0} \cdot x_{a} \cdot\left(\frac{Q_{X}(\tau)}{Q_{0}}\right)^{2-m}=Z_{K}+\frac{p_{K}(\tau)}{\rho \cdot g}+i_{L}(\tau) \cdot\left(L-x_{a}\right)
$$

Hence

$$
i_{L}(\tau)=\frac{\left(Z_{H}-Z_{K}\right)+\frac{p_{H}(\tau)-p_{K}(\tau)}{\rho \cdot g}-i_{0} \cdot x_{a} \cdot\left(\frac{Q_{X}(\tau)}{Q_{0}}\right)^{2-m}}{\left(L-x_{a}\right)}
$$

or

$$
i_{L}(\tau)=\frac{\left(Z_{H}-Z_{K}\right)}{\left(L-x_{a}\right)}+\frac{p_{H}(\tau)-p_{K}(\tau)}{\rho \cdot g\left(L-x_{a}\right)}-i_{0} \frac{x_{a}}{\left(L-x_{a}\right)}\left(\frac{Q_{X}(\tau)}{Q_{0}}\right)^{2-m}
$$

Taking into account the dependence (11), $Q_{L}(\tau)$ flow rate (3) after the transformation can be rewritten as following:

$$
Q_{L}(\tau)=Q_{0} \cdot\left[\frac{\left(Z_{H}-Z_{K}\right)}{\left(L-x_{a}\right) \cdot i_{0}}+\frac{p_{H}(\tau)-p_{K}(\tau)}{\rho \cdot g\left(L-x_{a}\right) \cdot i_{0}}-\frac{x_{a}}{\left(L-x_{a}\right)}\left(\frac{Q_{X}(\tau)}{Q_{0}}\right)^{2-m}\right]^{\frac{1}{2-m}}
$$

or after transformation:

Then final amount of leakage in the $\mathrm{j}$ - th point of time

$$
Q_{Y}(\tau)=Q_{X}(\tau)-\left[\left(Z_{H}-Z_{K}+\frac{p_{H}(\tau)-p_{K}(\tau)}{\rho \cdot g}\right) \cdot \frac{Q_{0}^{2-m}}{\left(L-x_{a}\right) \cdot i_{0}}-\frac{x_{a}}{\left(L-x_{a}\right)} Q_{X}^{2-m}(\tau)\right]^{\frac{1}{2-m}}
$$

The amount of oil leaked from the pipe in a time $\tau$ can be determined:

$$
\mathrm{V}=\int_{0}^{\tau} Q_{Y}(\tau) d \tau
$$

or using integral algorithm rectangles approximate formula for calculation: 


$$
\mathrm{V}=\sum_{j=\mathbf{1}}^{N} Q_{Y}^{j}(\tau) \Delta \tau
$$

where $\Delta \tau=\frac{\tau}{N}-$ approximate integration step, it is constant in this case; $\tau$ - the total duration of the expiration time of the product; $\mathrm{N}$ - the number of partitions of $\tau$ time on intervals $\Delta \tau$.

The amount of oil flowing out of the pipe can be found using the approximations method, for which, increasing $\mathrm{N}$ by one, the calculation $\mathrm{V}$ is carried out as long as it does not meet the following condition:

$$
|V N-1-V N| \leq \delta \cdot V N
$$

where $\delta$ - is a defined calculation error. For practical solutions it is enough to take $\delta=$ 0.05 .

The method was tested on the main oil pipeline with the use of specially prepared programs for PC and route profile [11-14, 21]. The results of experiments carried out on the existing pipeline with a diameter of $1220 \mathrm{~mm}$ can be considered the most complete and sufficiently representative (Figure 3) [22-23].

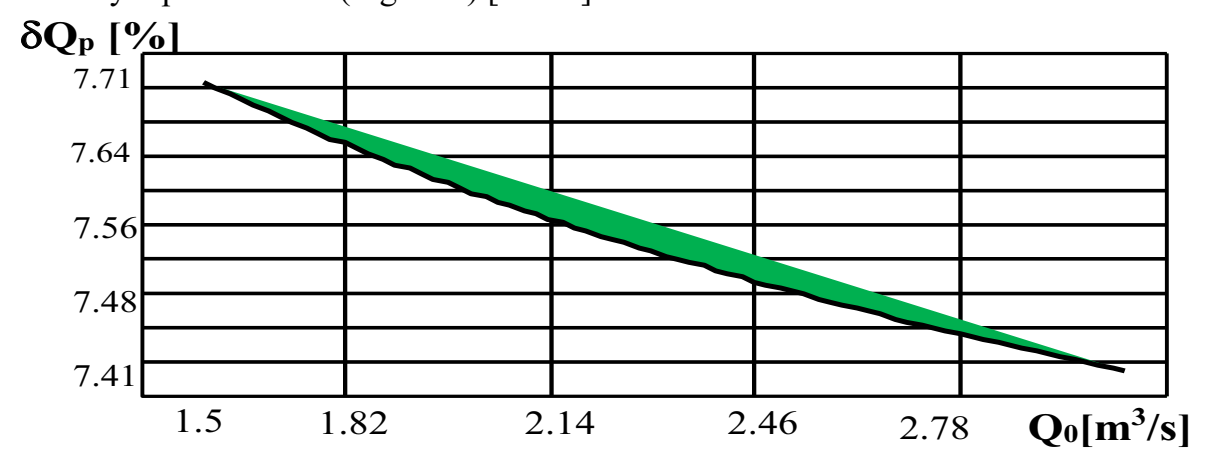

Fig. 3. The dependence of the inaccuracy $Q_{p}$ from the rate $Q_{0}$ to the pipeline of diameter $D=$ $1220 \mathrm{~mm}$.

\section{Conclusions}

Development and application of different methods of emission registration, determining the location and magnitude of leaks are caused by different degrees of complexity of the pipeline route, and the various conditions of its bedding in the soil [24]. As far as the detection of imperfections of the method used there is a necessity to find new, more accurate methods of control of the leaks, especially those that are hidden. Thus, the obtained dependence (16) allows determining the amount of leakage in the period when the pumping stations work over the damaged section of the pipeline based on the dispatching data on changes in pump station discharge pressure. In IUT calculation programs are developed. 


\section{References}

1. A technique by definition of damage to surrounding environment at accidents on the main oil pipelines. Ministry of Fuel and Energy (Transpress, Moscow, 1996)

2. V. Antipyev, Yu. Zemenkov, N. Kudryavtseva, UMN Z and SZS (Tyumen, 1985)

3. V. Kurushina, Yu. Zemenkov, WIT Transactions on Ecology and the Environment 190(2), 881-888 (2014)

4. V. Antip'ev, A. Nevolin, Yu. Zemenkov, Neftyanoe Khozyaistvo - Oil Industry 10, 4648 (1981)

5. V. Antipyev, V. Arkhipova, Yu. Zemenkov, N. Kudryavtseva, The instruction on calculation of losses of oil at refusals on the main oil pipelines of the Western and Northwest Siberia (TSOGU, Tyumen, 1986)

6. S. Dudin, Yu. Zemenkov, A. Shabarov, N. Saranchyn, Higher Educational Institutions News. Oil and Gas 6, 63-68 (2010)

7. A. Shipovalov, Yu. Zemenkov, S. Toropov, M. Zemenkova, S. Podorozhnikov, I. Tyrylgin, V. Pavlov, Aspects of technological reliability and economic efficiency of operation of underground storages of natural gas of Western Siberia (TSOGU, Tyumen, 2012)

8. Yu. Zemenkov, V. Shalay, M. Zemenkova, Procedia Engineering 113, 254-258 (2015)

9. Yu. Zemenkov, V. Shalay, M. Zemenkova, Procedia Engineering 113, 312-315 (2015)

10. Yu. Zemenkov, Dissertation of Doctor of Technical Sciences (Tyumen: TSOGU, 1999)

11. S. Stepanov, N., Cheremisin, S., Sokolov, A., Altunin, A., Shabarov, ECMOR 2012 13th European Conference on the Mathematics of Oil Recovery (2012)

12. S. Kudakaev, F. Aminev, V. Galiakbarov, G. Korobkov, V. Kovshov, Gas Industry 5, 10 (2004)

13. M. Lurie, Neftyanoe khozyaystvo - Oil Industry 9, 111-113 (2013)

14. M. Lurie, DOI: 10.1002/9783527626199 (2009)

15. E. Kurushina, V. Kurushina, Life Science Journal 11(11), 517-521 (2014)

16. V. Kurushina, Yu. Zemenkov, E. Kurushina, Fundamental researches 2-8, 1632-1636 (2015)

17. E. Kurushina, Canadian Journal of Science, Education and Culture 2(6), 378-384 (2014)

18. E. Kurushina, Problems of formation of a common economic space and social development in the CIS countries: Proceedings of the International scientific and practical conference (TSOGU, Tyumen. 2011)

19. S. Toropov, Yu. Zemenkov, S. Podorozhnikov, Gas industry 720(720), 95-98 (2015)

20. M. Zemenkova, L. Fayzulina, I. Seroshtanov, Gas industry 720(720), 59-64 (2015)

21. A. Shabarov, A. Ibragimov, M. Zemenkova, S. Podorozhnikov, Gas industry 12(715), 50-54 (2014)

22. A. Pimnev, M. Zemenkova, Pipeline transport: theory and practice 5(51), 43-47 (2015)

23. M. Zemenkova, Yu. Zemenkov, A. Pimnev, V. Petryakov, Business magazine Neftegaz.RU 11-12, 64-70 (2015)

24. R. Mamadaliev, V. Kuskov, Yu. Zemenkov, A. Popova, Applied Mechanics and Materials 770, 19-22 (2015) 